\title{
Fast Initialization of Cognitive Radio Systems
}

\author{
Malcolm Egan ${ }^{1}$, Jean-Marie Gorce ${ }^{1}$ and Leonardo Cardoso ${ }^{1}$ \\ ${ }^{1}$ CITI Lab, INRIA, INSA Lyon, Univ. Lyon, France
}

\begin{abstract}
Fast initialization of cognitive radio systems is a key problem in a variety of wireless communication systems, particularly for public safety organizations in emergency crises. In the initialization problem, the goal is to rapidly identify an unoccupied frequency band. In this paper, we formalize the initialization problem within the framework of active hypothesis testing. We characterize the optimal scanning policy in the case of at most one free band and show that the policy is computationally challenging. Motivated by this challenge for the implementation of the optimal policy and the need to cope with an unknown number of interferers larger than one, we propose the constrained DGF algorithm. We show that for strict constraints on the maximum number of observations, the constrained DGF algorithm can outperform the error probability of the stateof-the-art C-SPRT algorithm by an order of magnitude, for comparable average delays.
\end{abstract}

\section{INTRODUCTION}

Cognitive radio is now an established means of supporting coexistence of wireless communication systems operating on the same frequency bands, in the same region. Beyond improving spectral efficiency in cellular networks [1], cognitive radio is also recognized as a key technology in a range of other networks. For instance, a dedicated public safety wireless network of an organization (e.g., police, fire and medical) supporting communication on dedicated and unlicensed bands during an emergency crisis can suffer interference from other organizations [2]. Due to the clear need for each organization to reliably communicate, it is necessary to limit the interorganization interference. In particular, it is highly desirable for new organizations assisting in an emergency to initialize their communication networks to avoid introducing interference for existing organizations. As such, the initialization of each organization's wireless network plays an important role in supporting an effective emergency response.

The initialization problem for cognitive radio systems is closely related to the problem of efficiently scanning spectrum to find unoccupied frequency bands. To date, a number of spectrum scanning algorithms have been proposed based on techniques from statistical detection theory [3]. In the special case where a single band is observed, a large number of detection schemes have been developed based on the structure of the interfering wireless signals, such as energy [4], second order statistics [5] and cyclostationarity [6]. A key assumption underlying these schemes is a fixed number of observations.

However, in the initialization problem with multiple bands, there are two aspects not addressed by schemes for single band observation with a fixed number of samples. The first aspect is the need to minimize the number of samples before a band is identified as unoccupied, subject to a probability of error. To deal with this aspect, sequential detection algorithms originating in the work of Wald [7] and Page [8] have been proposed to identify when it is possible to stop observing and use only a small number of samples to meet error probability constraints. A key technique is sequential detection of the occupancy of a single band based on the sequential probability ratio test (SPRT) [7].

The second aspect is the ability to identify a free band within a larger set, when only a small number of bands can be observed simultaneously. Existing work has focused on the problem of identifying the occupancy of every band, where sequential hypothesis testing frameworks have been developed in [9], [10]. In [9], an optimal algorithm was proposed based on CUSUM [8]. This was extended in [10] to account for a constraint on the total number of observations. The detection problem was shown to be equivalent to a Markov optimal stopping problem; however, it was found that the optimal policy is computationally challenging. Motivated by the optimal scanning policy without a constraint on the number of observations, the truncated concatenated SPRT (C-SPRT) algorithm was proposed to provide a tradeoff between obtaining a low error probability and a practical computational complexity. However, the key challenge in applying this existing work to the initialization problem is that the focus is on identifying the occupancy state of all frequency bands, rather than minimizing the detection time of a single unoccupied band.

In this paper, we consider the initialization problem for cognitive radio based on minimizing the detection time of a single unoccupied band. The first step is to formalize the problem, where we adopt the Bayesian approach [11]. The formalization builds on the active hypothesis testing framework developed in [11], [12] for the purpose of anomaly detection. A key difference is that we allow for the possibility that no band is occupied and the presence of constraints on the maximum number of observations, which are important aspects of real-world cognitive radio systems. Our first result is a characterization of the optimal scanning policy when it is known that there is at most one free band. Using a dynamic programming formulation, we show that this policy is equivalent to the solution of a Markov optimal stopping problem. As also occurs in the optimal policy in [10] when identifying the occupancy state of all bands, the optimal policy relies on a computationally challenging recursion. As the difficulties for the optimal policy arise even for at most one free band, it suggests that the optimal policy is unlikely to be practical for an unknown number of free bands.

Motivated by the computational challenges of the optimal 
scanning policy, we propose the constrained DGF algorithm. The proposed algorithm is a heuristic low-complexity algorithm, which has a simple scanning rule depending only on the sum likelihood ratio at each time step. Moreover, the algorithm can be applied even when there is an unknown number of free bands, including zero.

We investigate the error probability and average delay of the constrained DGF algorithm numerically via Monte Carlo simulations in the case of zero mean Gaussian interferers and noise. In particular, we demonstrate the effect of algorithm parameters such as the sensing cost on the tradeoff between the error probability and the average delay. We then compare our algorithm with the C-SPRT algorithm proposed in [10] and show that the constrained DGF algorithm can outperform the error probability of the C-SPRT algorithm by an order of magnitude for strict constraints on the number of observations.

\section{Problem Setup}

\section{A. System Model}

Consider a base station, which aims to find a single frequency band in the set $\{1,2, \ldots, K\}$ that is unoccupied. We assume that the base station can observe a single band at a time. Let $Y_{k}^{(j)}$ be the signal sample observed by the base station, at time $j$ from the band $k$. If there are no transmissions by any other networks on band $k$, then the signal is

$$
Y_{k}^{(j)}=W_{k}^{(j)}
$$

where $W_{k}^{(j)}$ is noise. On the other hand, if there is a transmission on band $k$ at time $j$, then

$$
Y_{k}^{(j)}=X_{k}^{(j)}+W_{k}^{(j)},
$$

where $X_{k}^{(j)}$ is the interfering signal. If an interferer is present on band $k$ at time $j$, then the interferer is also present on band $k$ at any other time $j^{\prime}$. For each band $k$, the signal $Y_{k}^{(j)}$ has a probability density function, $f_{k}$, initially unknown to the base station. In general, the density $f_{k}$ will not be the same for each band. As such, we identify a set of densities $\mathcal{D}_{\text {free }}$ which correspond to free bands and a set of densities $\mathcal{D}_{\text {occ }}$ which correspond to occupied bands.

Given $n_{k}$ observations of band $k$, the base station can perform a hypothesis test in order to determine whether or not there is an interferer. In particular,

$$
\begin{aligned}
& H_{0}^{(k)}: Y_{k}^{(j)}=W_{k}^{(j)}, j=1,2, \ldots, n_{k} \\
& H_{1}^{(k)}: Y_{k}^{(j)}=X_{k}^{(j)}+W_{k}^{(j)}, j=1,2, \ldots, n_{k}
\end{aligned}
$$

where $H_{0}^{(k)}$ is the hypothesis that channel $k$ is unoccupied and $H_{1}^{(k)}$ is the hypothesis that channel $k$ is occupied. In the initialization problem, it is necessary to also choose which channels to sense. This affects the number of observations of band $k, n_{k}$, available to make the decision of whether or not the band is occupied. As it is necessary to complete initialization within a short period of time, a constraint on the maximum number of observations is required. In particular, at each time $n$

$$
\sum_{k=1}^{K} n_{k}=n \leq T
$$

\section{B. Problem Formulation}

Our goal is to identify the first unoccupied band $k \in$ $\{1,2, \ldots, K\}$; i.e., the band with the lowest index such that the probability density function $f_{k} \in \mathcal{D}_{\text {free }}$. If band $k$ is the first unoccupied band, then hypothesis $H_{k}^{(0)}$ is said to be true. We adopt a Bayesian approach and assign an a priori probability that $H_{k}^{(0)}$ is true, denoted by $\pi_{k}$. As it is possible that no bands lie in $\mathcal{D}_{\text {free }}$, we also consider a band 0 such that $H_{0}^{(0)}$ is true if $H_{k}^{(0)}$ is not true for all $k \in\{1,2, \ldots, M\}$. The a priori probabilities $\pi_{k}, k \in\{0,1,2 \ldots, K\}$ satisfy $\sum_{k=0}^{K} \pi_{k}=1$

At each time, the base station can observe a single band. When the null hypothesis $H_{k}^{(0)}$ is true for band $k$, the probability density function of $Y_{k}^{(j)}$ lies in the set $\mathcal{D}_{\text {free }}$. On the other hand, if $H_{k}^{(0)}$ is false, there is an interferer and $f_{k}$ lies in $\mathcal{D}_{\text {occ }}$.

The time at which the base station stops scanning is denoted by $\tau$. At time $\tau$, the base station declares a band $k \in\{1,2, \ldots, K\}$ as free or declares that no band is free by reporting $k=0$. The base station's decision rule is denoted by $\delta$, with $\delta \in\{0,1,2, \ldots, K\}$. At each time $n$, the base station makes a decision for which band to scan. The selection rule is denoted by $\phi(n) \in\{1,2, \ldots, K\}$, indicating the band to be scanned in time slot $n$.

With a finite number of observations, it is not possible to be certain that any given band lies in $\mathcal{D}_{\text {free }}$ due to the presence of noise. Let $\alpha_{k}=\mathbb{P}_{k}(\delta \neq k)$ be the probability of declaring $\delta \neq$ $k$ when band $k$ is in fact the first band that lies in $\mathcal{D}_{\text {free }}$. The probability of error is then defined by $P_{e}=\sum_{k=0}^{M} \pi_{k} \alpha_{k}$. This notion of error forms an upper bound on the total probability of error, which is the probability that a band $k$ is selected with $f_{k} \in \mathcal{D}_{\text {occ }}$ or band $k=0$ is selected when there is a free band. We consider the probability of error as it provides a tractable yet practically useful means of obtaining characterizations of the optimal policy. Moreover, it is desirable to find free bands with low indices, particularly if more than one base station is scanning at the same time. We investigate the total probability of error via simulations in Section V.

The other key factor in the initialization problem is the detection delay. To this end, consider the average detection delay given hypothesis $H_{0}^{(k)}$ is true, denoted by $\mathbb{E}_{k}[\tau]$. The average detection delay is then $\mathbb{E}[\tau]=\sum_{k=0}^{K} \mathbb{E}_{k}[\tau]$.

For each observation there is a sensing cost, which is denoted by $c \in \mathbb{R}_{+}$. The Bayes risk when hypothesis $H_{k}^{(0)}$ is true is then given by

$$
R_{k}=\alpha_{k}+c \mathbb{E}_{k}[\tau]
$$


and the average Bayes risk is

$$
R=\sum_{k=0}^{K} R_{k}=P_{e}+c \mathbb{E}[\tau] .
$$

The initialization problem is therefore equivalent to finding a policy consisting of a stopping time $\tau$, a decision rule $\delta$ and a band selection rule $\phi(n), n=1,2, \ldots, T$ that minimizes the average Bayes risk $R$.

\section{Optimal Scanning Policies}

In order to gain insight into how to design an initialize algorithm, we first characterize the optimal scanning policy when at most one band is free and $\mathcal{D}_{\text {free }}=\left\{f_{0}\right\}, \mathcal{D}_{\text {occ }}=\left\{f_{1}\right\}$ such that $\left|\mathcal{D}_{\text {free }}\right|=\left|\mathcal{D}_{\text {occ }}\right|=1$. An example of this scenario occurs is when the noise, $W_{k}^{(j)}$, is zero mean Gaussian random variable with a known variance $\sigma_{W}^{2}$ and the signal, $X_{k}^{(j)}$ is also zero mean Gaussian random variable with a known variance $\sigma_{X}^{2}$. We show that even in this restricted scenario, the optimal policy is computationally challenging and not ideal for practical implementation.

Let $q_{i}^{k}(z)$ be the probability density function of an observation on band $z$ given that band $i$ is free. In particular,

$$
q_{i}^{k}(z)= \begin{cases}f_{0}(z), & i \neq k \\ f_{1}(z), & i=k .\end{cases}
$$

Consider the Bayesian update of the belief vector $\pi^{(n)}=$ $\left[\pi_{0}^{(n)}, \pi_{1}^{(n)}, \ldots, \pi_{K}^{(n)}\right]$ by the $n_{k}$-th observation of band $k$, $Y_{k}^{\left(n_{k}\right)}$, given by

$$
\begin{aligned}
& \Phi_{k}\left(\pi^{(n)}, Y_{k}^{\left(n_{k}\right)}\right) \\
& =\left[\pi_{0}^{(n-1)} \frac{q_{0}^{k}\left(Y_{k}^{\left(n_{k}\right)}\right)}{q_{\pi^{(n-1)}}^{k}\left(Y_{k}^{\left(n_{k}\right)}\right)}, \ldots, \pi_{K}^{(n-1)} \frac{q_{K}^{k}\left(Y_{k}^{\left(n_{k}\right)}\right)}{q_{\pi^{(n-1)}}^{k}\left(Y_{k}^{\left(n_{k}\right)}\right)}\right],
\end{aligned}
$$

where $q_{\pi^{(n-1)}}^{k}\left(Y_{k}^{\left(n_{k}\right)}\right)=\sum_{j=1}^{K} \pi_{j}^{(n-1)} q_{j}^{k}\left(Y_{k}^{\left(n_{k}\right)}\right)$. Define the cost-to-go function given a filtration $\mathcal{F}_{n}$ (interpreted as the information available at time $n$ ) as

$$
\tilde{R}\left(\mathcal{F}_{n}\right)=c+\min _{k=1,2, \ldots, K} \mathbb{E}\left[\tilde{R}_{n+1}\left(\mathcal{F}_{n+1} \mid k, \mathcal{F}_{n}\right)\right] .
$$

The value function can then be defined recursively as

$$
\begin{array}{r}
R=\min \left\{\min _{k=1, \ldots, K} c+\mathbb{E}\left[\tilde{R}_{n+1}\left(\mathcal{F}_{n+1} \mid k, \mathcal{F}_{n}\right)\right],\right. \\
\left.\min _{k=1,2, \ldots, K} 1-\pi_{k}^{\left(n_{k}\right)}\right\},
\end{array}
$$

We now show that $\pi^{(n)}$ is a sufficient statistic capturing the past history of the process, which forms a basis to characterize the optimal policy. To begin, the cost-to-go at time $T$ is

$$
\tilde{R}_{T}\left(\mathcal{F}_{n}\right)=1-\pi_{k^{*}}^{(T)}
$$

where $k^{*}=\min _{k=0,1,2, \ldots, M} 1-\pi_{k}^{(T)}$. For $j<T$, the cost-togo is given by $\tilde{R}$ in (10), where $\min _{k=0,1,2, \ldots, K} 1-\pi_{k}^{(n)}$ is the cost incurred if scanning is terminated and the expected cost of continuing scanning is given by

$$
\min _{k=1,2, \ldots, K} c+\mathbb{E}\left[\tilde{R}_{n+1}\left(\mathcal{F}_{n+1}\right) \mid k, \mathcal{F}_{n}\right] .
$$

Note that the expectation $\mathbb{E}\left[\tilde{R}_{n+1}\left(\mathcal{F}_{n+1}\right) \mid k, \mathcal{F}_{n}\right]$ depends on $\mathcal{F}_{n}$, which is the entire observation history so far.

Observe that $\tilde{R}_{T}$ only depends on $\pi^{(T)}$. We show that $\pi^{(n)}$ is also a sufficient statistic for $n<T$ using an argument based on induction. Suppose that $\tilde{R}_{n}\left(\mathcal{F}_{n+1}\right)$ depends on $\pi^{(n+1)}$ only, which we denote by $\hat{R}_{n+1}\left(\pi^{(n+1)}\right)$. Then,

$$
\begin{aligned}
\tilde{R}\left(\mathcal{F}_{n}\right)=\min \left\{c+\min _{k=1,2, \ldots, K} \mathbb{E}\left[\tilde{R}_{n+1}\left(\mathcal{F}_{n+1} \mid k, \mathcal{F}_{n}\right)\right],\right. \\
\left.\min _{k=0,1, \ldots, K} 1-\pi_{k}^{(n)}\right\} \\
=\min \left\{c+\min _{k=1, \ldots, K} \mathbb{E}\left[\hat{R}_{n+1}\left(\pi^{(n+1)}\right) \mid k, \mathcal{F}_{n}\right],\right. \\
\left.\min _{k=0,1, \ldots, K} 1-\pi_{k}^{(n)}\right\} .
\end{aligned}
$$

We then have

$$
\begin{aligned}
& \mathbb{E}\left[\hat{R}_{n+1}\left(\pi_{n+1}\right) \mid k, \mathcal{F}_{n}\right] \\
& =\int R_{n+1}\left(\Phi_{k}\left(\pi^{(n)}, y\right)\right) q_{\pi^{(n)}}^{k}(y) d y \\
& =A_{n}^{(k)}\left(\pi^{(n)}\right) .
\end{aligned}
$$

It then follows that

$$
k^{*}=\underset{k=1,2, \ldots, K}{\operatorname{argmin}} A_{n}^{(k)}\left(\pi^{(n)}\right) .
$$

As such $\pi^{(n)}$ is a sufficient statistic. Moreover, since the choice of $k$ only depends on $\pi^{(n)}$, the process $\left\{\pi^{(n)}, n=0,1,2, \ldots\right\}$ is Markov.

Using the same argument as in [13, Theorem 1], $\mathbb{E}\left[\hat{R}\left(\pi^{(n+1)}\right) \mid k, \pi^{(n)}\right]$ is concave in $\pi^{(n)}$ for all $k=$ $1,2, \ldots, K$. This implies that the cost-to-go $\tilde{R}\left(\mathcal{F}_{n}\right)$ is concave and the optimal policy is the solution to a Markov optimal stopping time problem. The optimal stopping rule is therefore [14]

$$
\begin{array}{r}
\tau=\inf \left\{n: \min _{k=0,1, \ldots, K} c+\mathbb{E}\left[\hat{R}_{n+1}\left(\pi_{n+1} \mid k, \pi_{n}\right)\right] \geq\right. \\
\left.\min _{k=1,2, \ldots, K} 1-\pi_{k^{*}}^{n}\right\} .
\end{array}
$$

To summarize, we have shown that the initialization problem in this scenario is equivalent to a Markov optimal stopping time problem. However, computing the policy to select a band to observe relies on the recursion in (15). To reduce the computational requirements and cope with more general scenarios allowing for an unknown number of free bands larger than one, it is desirable to consider simpler policies. 


\section{Proposed Initialization Algorithm}

In this section, we propose an initialization algorithm to efficiently find a single unoccupied band. Motivated by the complexity of the optimal scanning policy characterized in Section III, the decision of which band to sense and the stopping criterion rely on a condition that is straightforward to verify. In particular, the algorithm is based on the DGF algorithm [11] developed for anomaly detection within the active hypothesis testing framework of Chernoff [12].

Let $f_{r, i} \in \mathcal{D}_{\text {free }}$ be a probability density function in $\mathcal{D}_{\text {free }}$ and $f_{r, o}$ be a probability density function in $\mathcal{D}_{o c c}$. At time $n$, define $\Lambda_{n}^{\left(k_{l}\right)}$ as the $l$-th largest sum likelihood (corresponding to the band with index $k_{l}$ ) given by

$$
\Lambda_{n}^{\left(k_{l}\right)}=\sum_{j=1}^{n_{k_{l}}} \frac{f_{r, i}\left(Y_{k_{l}}^{\left(n_{k_{l}}\right)}\right)}{f_{r, o}\left(Y_{k_{l}}^{\left(n_{k_{l}}\right)}\right)} .
$$

The constrained DGF algorithm is detailed as follows.

1) Initialization: Select $c>0, f_{r, i} \in \mathcal{D}_{\text {free }}, f_{r, o} \in \mathcal{D}_{o c c}$, and set $n=1$.

2) Loop: For each time $n$, select a band $k$ according to the rule

$$
k= \begin{cases}k_{1}, & D\left(f_{r, i} \| f_{r, o}\right) \geq \frac{D\left(f_{r, o} \| f_{r, i}\right)}{K-1} \\ k_{2}, & D\left(f_{r, i} \| f_{r, o}\right)<\frac{D\left(f_{r, o} \| f_{r, i}\right)}{K-1},\end{cases}
$$

where $D(\cdot \| \cdot)$ is the Kullback-Leibler divergence [15].

3 ) Based on the new observation of band $k$, update the ordering of the sum likelihoods $\left\{\Lambda_{n}^{(k)}\right\}_{k=1}^{K}$. If $\Lambda_{n}^{(1)} \geq$ $-\log c$, then terminate and set $k=k_{1}$. Similarly, if $n+1>T$, then terminate and set $k=0$. Otherwise, repeat Step 1 for time $n+1$.

The constrained DGF algorithm has several desirable features. First, the band selection rule in time slot $n$ given in (19) is simple, particularly compared with the optimal policy in Section III. Second, the constrained DGF algorithm can be applied when there is an unknown number $(\geq 1)$ of unoccupied bands and when $\left|\mathcal{D}_{\text {free }}\right|>1$ or $\left|\mathcal{D}_{\text {occ }}\right|>1$. Third, when the only element of $\mathcal{D}_{\text {free }}$ is $f_{r, i}$ and the only element of $\mathcal{D}_{\text {occ }}$ is $f_{r, o}$ and it is known that there is only a single free band, then as $c \rightarrow 0$ it follows from [11, Theorem 1] that

$$
R \sim \frac{-c \log c}{D\left(f_{r, o} \| f_{r, i}\right)},
$$

which agrees with the Bayes risk minimizing policy.

However, to apply the constrained DGF algorithm it is necessary to select the sensing cost $c$. In the following section, we investigate the effect of these parameters on the total error probability and average delay of the algorithm and compare it with the C-SPRT algorithm proposed in [10].

\section{NumericAl RESUlts}

In this section, we numerically evaluate the total error probability and average delay of the constrained DGF algorithm for zero-mean Gaussian signal and noise with variances $\sigma_{X}^{2}$ and $\sigma_{W}^{2}$, respectively. In particular,

$$
\begin{aligned}
& f_{r, i}(x)=\frac{1}{\sqrt{2 \pi \sigma_{W}^{2}}} \exp \left(-\frac{x^{2}}{2 \sigma_{W}^{2}}\right) \\
& f_{r, o}(x)=\frac{1}{\sqrt{2 \pi\left(\sigma_{X}^{2}+\sigma_{W}^{2}\right)}} \exp \left(-\frac{x^{2}}{2\left(\sigma_{W}^{2}+\sigma_{X}^{2}\right)}\right) \text {. }
\end{aligned}
$$

To implement the constrained DGF algorithm, the KullbackLeibler divergences in (19), which are given by

$$
\begin{aligned}
& D\left(f_{r, i} \| f_{r, o}\right)=\log \left(\frac{\sigma_{W}^{2}+\sigma_{X}^{2}}{\sigma_{W}^{2}}\right)+\frac{\sigma_{W}^{2}}{2\left(\sigma_{W}^{2}+\sigma_{X}^{2}\right)}-\frac{1}{2} \\
& D\left(f_{r, o} \| f_{r, i}\right)=\log \left(\frac{\sigma_{W}^{2}}{\sigma_{W}^{2}+\sigma_{X}^{2}}\right)+\frac{\sigma_{W}^{2}+\sigma_{X}^{2}}{2 \sigma_{W}^{2}}-\frac{1}{2}
\end{aligned}
$$

In the simulations, $K=7$, with each band unoccupied with probability 0.1 . The signal-to-noise ratio $\sigma_{X}^{2} / \sigma_{W}^{2}$ is $-5 \mathrm{~dB}$.

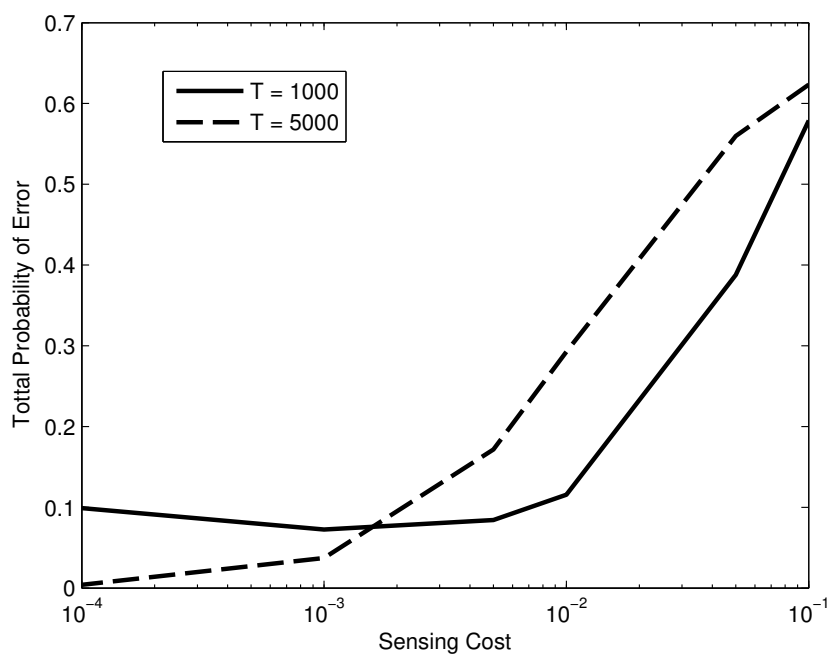

Fig. 1. The effect of the sensing cost, $c$, on the total error probability for varying observation constraint, $T$.

In Fig. 1 and Fig. 2, we consider the effect of the observation constraint, $T$, and the sensing cost, $c$. Fig. 1 shows the total error probability as the sensing cost varies, for $T=1000$ and $T=5000$. Observe that for sufficiently large sensing cost $c$, the total error probability decreases for decreasing $c$. This is due to the fact that for large $c$, the sensing cost reduces the number of observations and increases the total error probability. There are two other key observations. First, a larger $T$ does not imply that the total error probability is lower for all choices of the sensing cost. This is due to an improved identification of the case that there are no unoccupied bands. Second, the total error probability is increasing for low values of $c$ with $T=1000$. This arises because decreasing $c$, leads to a larger number of observations exceeding $T$ which means that free bands are incorrectly identified as occupied.

Fig. 2 shows that average delay as the sensing cost varies. Observe that increasing the sensing cost reduces the average delay. This is because a high sensing cost causes the constrained DGF algorithm to terminate faster than for a low sensing cost. 


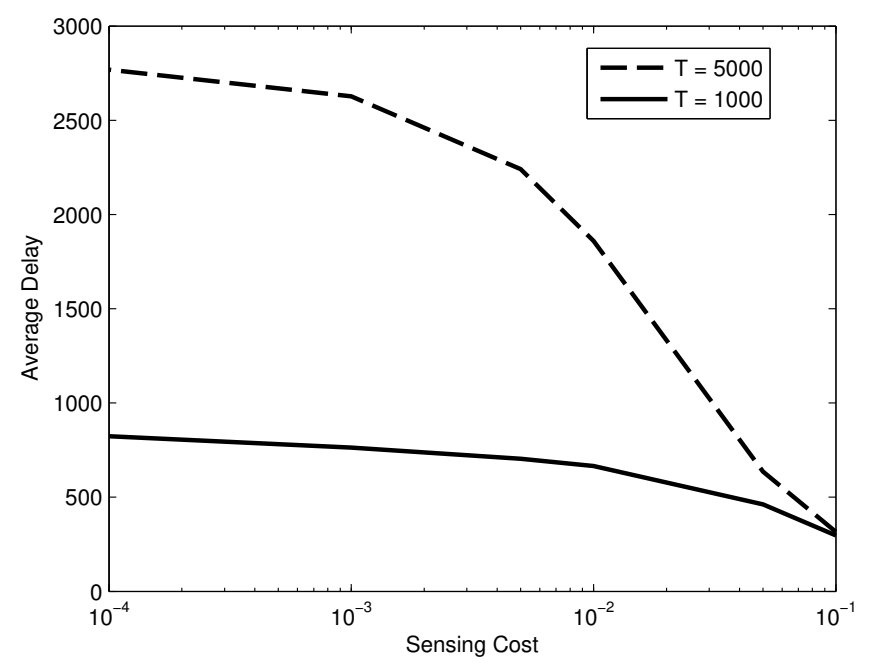

Fig. 2. The effect of the sensing cost, $c$, on the average delay for varying observation constraint, $T$.

TABLE I

COMPARISON OF THE CONSTRAINED DGF AND C-SPRT ALGORITHMS

\begin{tabular}{|c|c|c|c|}
\hline & $\mathrm{T}$ & Average Delay & Total Error Probability \\
\hline Cons. DGF & 1000 & 705.6 & 0.085 \\
C-SPRT & 1000 & 839.9 & 0.404 \\
\hline Cons. DGF & 2000 & 1107.1 & 0.11 \\
C-SPRT & 2000 & 1092.1 & 0.17 \\
\hline Cons. DGF & 5000 & 1104.3 & 0.349 \\
C-SPRT & 5000 & 1157.7 & 0.091 \\
\hline
\end{tabular}

Table I compares the proposed constrained DGF algorithm with the C-SPRT algorithm proposed in [10] which is modified to terminate after the first unoccupied channel is identified. The experiments compare the total error probability with comparable average delays. Observe that for a maximum of $T=1000$ observations, the constrained DGF algorithm leads to an order of magnitude reduction in the total error probability. For an increasing maximum number of observations, the C-SPRT algorithm begins to outperform the constrained DGF algorithm above $T=2000$ observations. The reason is that the C-SPRT algorithm is able to identify the scenario where all bands are unoccupied more rapidly than the constrained DGF algorithm, for large values of $T$. This suggests that an adaptive approach using the constrained DGF algorithm for small values of $T$ and C-SPRT for large values of $T$ is an effective solution to the initialization problem.

\section{Conclusions}

Initialization of cognitive radio systems plays an important role for public safety organizations seeking to communicate in an emergency crisis. In particular, a key challenge is to quickly identify an unoccupied frequency band with a low probability of error. In this paper, we formalize the initialization problem within the framework of active hypothesis testing. We characterize the optimal spectrum scanning policy when there is at most one free band and show that a computationally challenging recursion must be solved. Motivated by this and the need to allow for an unknown number of unoccupied bands greater than one, we proposed the constrained DGF algorithm. We demonstrated the effect of the sensing cost parameter and the constraint on the maximum number of observations via Monte Carlo simulations. Moreover, we compared the algorithm with the existing C-SPRT algorithm designed to identify the occupancy state of all bands. A key conclusion is that for strict constraints on the maximum number of observations, the proposed constrained DGF algorithm can outperform the C-SPRT algorithm by an order of magnitude. We also found that for a large maximum number of observations, $T$, the C-SPRT algorithm is a better choice suggesting that an adaptive approach using the constrained DGF algorithm for small values of $T$ and the C-SPRT algorithm for large values of $T$ is an effective strategy.

\section{ACKNOWLEDGEMENTS}

This work was partially funded by the French PIA FED4PMR project.

\section{REFERENCES}

[1] X. Hong, J. Wang, C.-X. Wang, and J. Shi, "Cognitive radio in 5G: a perspective on energy-spectral efficiency trade-off," IEEE Communications Magazine, vol. 52, no. 7, pp. 46-53, 2014.

[2] G. Baldini, S. Karanasios, D. Allen, and F. Vergari, "Survey of wireless communication technologies for public safety," IEEE Communication Surveys and Tutorials, vol. 16, no. 2, pp. 619-641, 2013.

[3] E. Axell, G. Leus, and H. Poor, "Spectrum sensing for cognitive radio: state-of-the-art and recent advances," IEEE Signal Processing Magazine, vol. 29, no. 3, pp. 101-116, 2012.

[4] F. Digham, M.-S. Alouini, and M. Simon, "On the energy detection of unknown signals over fading channels," IEEE Transactions on Communications, vol. 55, no. 1, pp. 21-24, 2007.

[5] E. Axell and E. Larsson, "Optimal and sub-optimal spectrum sensing of OFDM signals in known and unknown noise variance," IEEE Journal of Selected Areas of Communication, vol. 29, pp. 290-304, 2011.

[6] J. Lundén, S. Kassam, and V. Koivunen, "Robust nonparametric cyclic correlation-based spectrum sensing for cognitive radio," IEEE Transactions on Signal Processing, vol. 58, no. 1, pp. 38-52, 2009.

[7] A. Wald, "Sequential tests of statistical hypotheses," The Annals of Mathematical Statistics, vol. 16, no. 2, pp. 117-186, 1945.

[8] E. Page, "Continuous inspection schemes," Biometrika, vol. 41, no. 1/2, pp. 100-115, 1954.

[9] L. Lai, H. Poor, Y. Xin, and G. Georgiadis, "Quickest search over multiple sequences," IEEE Transactions on Information Theory, vol. 57, no. 8, pp. 5375-5386, 2011.

[10] R. Caromi, Y. Xin, and L. Lai, "Fast multiband spectrum scanning for cognitive radio systems," IEEE Transactions on Communications, vol. 61, no. 1, pp. 63-75, 2013.

[11] K. Cohen and Q. Zhao, "Active hypothesis testing for anomaly detection," IEEE Transactions on Information Theory, vol. 61, no. 2, pp. 1432-1450, 2015.

[12] H. Chernoff, "Sequential design of experiments," Annals of Mathematical Statistics, vol. 30, no. 3, pp. 755-770, 1959.

[13] M. Naghshvar and T. Javidi, "Active M-ary sequential hypothesis testing," in IEEE International Symposium on Information Theory (ISIT), 2010 .

[14] A. Shiryaev, Optimal Stopping Rules. Springer, 2008.

[15] T. Cover and J. Thomas, Elements of Information Theory, Second Edition. John Wiley and Sons, Inc., 2006. 